Journal of Mathematical Physics, Analysis, Geometry

2014, vol. 10, No. 2, pp. 221-232

\title{
Smarandache Theorem in Hyperbolic Geometry
}

\author{
A.V. Kostin \\ Yelabuga Branch of the A.N.Tupolev National Technical University of Kazan \\ 16 Stroiteley Str., Yelabuga 423602, Russia \\ E-mail: kostin_andrei@mail.ru \\ I.Kh. Sabitov \\ M.V. Lomonosov Moscow State University \\ GSP-1, Leninskiye Gory, Moscow 119991, Russia \\ E-mail: isabitov@mail.ru
}

Received July 12, 2013, revised February 23, 2014

In the paper a hyperbolic version of the Smarandache pedal polygon theorem is considered.

Key words: hyperbolic geometry, spherical geometry, pedal polygon.

Mathematics Subject Classification 2010: 51M09.

\section{A Statement and History of the Problem}

On the Euclidean plane, let be given a polygon $Q$ with cyclicly numbered vertices $A_{1}, A_{2}, \ldots, A_{n}$ and a point $M$ in an arbitrary position. Denote by $M_{k}$ the orthogonal projections of the point $M$ on the lines $A_{k} A_{k+1}, 1 \leq k \leq n, A_{n+1}=A_{1}$. The polygon $P$ with successive vertices $M_{1}, M_{2}, \ldots, M_{n}$ is called the pedal polygon of the point $M$ relatively to the polygon $Q$ (sometimes $P$ is called the pedal polygon of the polygon $Q$ relatively to the point $M$ ). About the pedal polygons of triangles one can read, for example, in [1], v.1, ch. 10. Among classical results on pedals, the following assertion, known as the second Carnot theorem, is of the most interest to us: The lengths of segments formed by successive vertices of a triangle and its pedal triangle satisfy the equality

$$
A_{1} M_{1}^{2}+A_{2} M_{2}^{2}+A_{3} M_{3}^{2}=M_{1} A_{2}^{2}+M_{2} A_{3}^{2}+M_{3} A_{1}^{2} .
$$

In 1983, in [2] Smarandache generalized the assertion below for general $n$-gons in the Euclidean plane.

The work of the second author is supported by the grant of Russia Government 11.G34.31.0053.

(c) A.V. Kostin and I.Kh. Sabitov, 2014 
Theorem 1. The lengths of segments formed by successive vertices of an $n$-gon and its pedal polygon satisfy the equality

$$
A_{1} M_{1}^{2}+A_{2} M_{2}^{2} \cdots+A_{n} M_{n}^{2}=M_{1} A_{2}^{2}+M_{2} A_{3}^{2} \cdots+M_{n} A_{1}^{2} .
$$

When we consider polygons on a sphere, then on the definition of the pedal polygon we should impose some additional conditions related with non-uniqueness of the orthogonal projection of the point $M$ in spherical lines in some cases. By this reason, in [3] the notion of a regular point on the sphere of radius $R$ is introduced (a point $M$ is called regular for a given spherical polygon if it is not the pole for any line containing an edge of the polygon, that is, the point $M$ does not coincide with the center of any great circle containing an edge of the polygon) and the following theorem is proved:

Theorem 2. Let the points $M_{k}, k=\overline{1, n}$ be orthogonal projections of a regular point $M$ on the sides $A_{k} A_{k+1}\left(A_{n+1}=A_{1}\right)$ of a polygon $A_{1} A_{2} \ldots A_{n}$ on the sphere of radius $R$. Then

$$
\cos \frac{A_{1} M_{1}}{R} \cos \frac{A_{2} M_{2}}{R} \ldots \cos \frac{A_{n} M_{n}}{R}=\cos \frac{M_{1} A_{2}}{R} \cos \frac{M_{2} A_{3}}{R} \ldots \cos \frac{M_{n} A_{1}}{R} .
$$

As for validity of this theorem for polygons and their pedals in the Lobachevsky plane, there are series of results: in [4] and [5] the generalization of the above Carnot theorem is given (it is also mentioned in [6], p. 31), and in [7] a variant of the generalization of the Smarandache theorem for convex polygons is given (in [6], p. 32, an analogue of this assertion is mentioned for the upper half-plane model but without any proof or reference). Apparently it should be referred to [8] found in Intellectual Archive. But the results of these works cannot be considered as a complete solution to the problem of the generalization of the Smarandache theorem to the Lobachevsky plane by the following reasons.

1. In all the papers referred to above it is underlined that the question is about the Smarandache theorem for the concrete interpretation of the Lobachevsky plane, Poincaré models in a circle or upper half-plane, but not for the Lobachevsky plane itself. This approach influences even on the formulation of the theorem and makes it more complicated by including complex coordinates of vertices. There is also some confusion between the validity of the theorem on the upper half-plane model and on the "abstract" Lobachevsky plane.

2. Considered is only the case of the convex polygons $Q$. Moreover, an additional assumption on the position of the point $M$ is set. Namely, it is supposed that triangles with the vertex $M$ and neighbouring geodesics $M_{k} M$ and $A_{j} M$, where $j=k$ or $j=k+1,1 \leq k \leq n$, should be situated in the interior of $Q$. For example, in the case of an obtuse triangle, no any point in the interior of $Q$ can be chosen as $M$. 
3. In the proof of the inverse theorem, a non-existing property of hyperbolic functions is used: in the right-hand side of the identity

$$
\cosh (\alpha) \cosh (\beta)=\frac{\cosh (\alpha+\beta)+\cosh (\alpha-\beta)}{2}
$$

the difference of cosh is written instead of the sum of cosh.

The purpose of our paper is to give the generalization of the Smarandache theorem to the Lobachevsky plane in a complete form, that is, without any restrictions set on the shape of a polygon and with a maximal extension of the positions of the point $M$, including its position on the absolute line or in the ideal domain with a convenable interpretation of the orthogonal projection of this point to the lines continuing the edges of the polygon. Notice that if the point $M$ is in the ideal domain, then we will require that their polar lines be divergent with all the lines containing the edges of the considered polygon (for more details see Section 6 below), and for the points $M$ on the absolute circle we exclude the case where the orthogonal projection on the sides of the polygon are on the absolute.

$\mathrm{R}$ e $\mathrm{m} \mathrm{a} \mathrm{r} \mathrm{k}$ 1. Our interest to the subject appeared while discussing the N. Sönmez's question from the letter [9] to the second author.

\section{Formulation of the Main Result}

First of all, let us specify the notion of a polygon in the Lobachevsky plane understanding it in the same sense as it is done in [10] for the Euclidean case. Namely, on a circle, let be given $n$ points $A_{1}^{*}, A_{2}^{*}, \ldots, A_{n}^{*}$, numerated in one of cyclic orders, and let a map $f$ from the set of these points to the Lobachevsky plane be given with a condition that the images of the neighbouring points $A_{k}=f\left(A_{k}^{*}\right)$ and $A_{k+1}=f\left(A_{k+1}^{*}\right)$ do not coincide. Join the images of any two neighbouring points $A_{k}$ and $A_{k+1}, 1 \leq k \leq n\left(A_{n+1}=A_{1}\right)$ by a segment of the straight line of the Lobachevsky plane. We will call the obtained figure an $n$-gon in the Lobachevsky plane; the points $A_{k}$ will be called the vertices of the polygon, and the segments joining neighbouring vertices will be called the sides or edges of the polygon (if the orientation is to be taken into account, we will suppose that it is in accordance with the numeration of vertices). From the condition $f\left(A_{k}^{*}\right) \neq f\left(A_{k+1}^{*}\right)$ it follows that the lengths of the sides are not equal to zero, but self-intersections and complete or partial superpositions of edges may occur.

The main theorem is as follows.

Theorem 3. In the Lobachevsky plane of the curvature $K=-\frac{1}{R^{2}}$ let be given an n-gon $P: A_{1} A_{2} \ldots A_{n}$ and a point $M$ in an arbitrary position, including also 
the position of $M$ in the absolute or in the ideal domain. Let $M_{1}, M_{2}, \ldots, M_{n}$ be orthogonal projections of the point $M$ in the lines $A_{k} A_{k+1}, 1 \leq k \leq n$, obtained by continuing the sides $A_{k} A_{k+1}$. Then the lengths of the segments, formed by successive vertices of $Q$ and its pedal polygon $P$ relatively to the point $M$, satisfy the equality

$$
\prod_{k=1}^{n} \cosh \frac{A_{k} M_{k}}{R}=\prod_{k=1}^{n} \cosh \frac{M_{k} A_{k+1}}{R} .
$$

R e m a r k 2. Equality (3) can also be written in another form

$$
\sum_{k=1}^{n} \ln \left(\cosh \frac{A_{k} M_{k}}{R}\right)=\sum_{k=1}^{n} \ln \left(\cosh \frac{M_{k} A_{k+1}}{R}\right) .
$$

$\mathrm{R}$ e $\mathrm{m}$ a $\mathrm{rk} 3$. By the general idea of the relation between the formulae of spherical and hyperbolic geometries, (3) is "naturally expected" from formula (2) by changing $R$ into $i R$. However, this method allows us to predict only the form of dependance and does not claim that its application always ensures the validity of the obtained result.

$\mathrm{R} \mathrm{e} \mathrm{m}$ a $\mathrm{r} \mathrm{k}$ 4. We remark that according to the projective interpretation of the Lobachevsky geometry, the perpendicular from the point $M$ to the line $A_{k} A_{k+1}$ passes through the pole of the line $A_{k} A_{k+1}$ respectively to the polaritet defined by the absolute, that is, by the point of intersection of tangents to the absolute at the end points of the line $A_{k} A_{k+1}$.

For all admissible positions of the point $M$, one can prove a theorem inverse in some sense to the main theorem. Its exact formulation will be given in Sec. 5 .

\section{The Extended Lobachevsky Plane}

Let us consider an oval quadric in the real projective plane as the absolute of a hyperbolic plane. The points in the interior of the quadric will be called proper points, the points in the quadric will be called infinite points, and the points out of the quadric will be called the ideal points of the extended Lobachevsky plane [11]. In the interior of the quadric, the Lobachevsky geometry is realized. In the exterior of the quadric we have the geometry of a constant curvature with an indefinite metric. The exterior domain is called the ideal domain of the Lobachevsky plane or the De Sitter plane. The projective transformations mapping the quadric onto itself are the motions in the hyperbolic and the De Sitter planes, respectively. The lines intersecting the absolute at two real points are called hyperbolic lines, those tangent to the absolute are called isotropic or parabolic lines, others not having any point in common with the absolute are 
called elliptic lines. The ideal domain of the Lobachevsky plane topologically is the Möbius band.

The Poincaré model with the metric

$$
d s^{2}=R^{2} \frac{d x^{2}-d y^{2}}{y^{2}}, y>0
$$

covers the whole ideal domain except one isotropic line. In this case, the absolute is the $x$-axe with one improper point. The curvature of this metric is $\frac{1}{R^{2}}$. In the considered Poincaré model of pseudo-Euclidean plane, the hyperbolic lines are real lines and semi-circles orthogonal to the $x$-axe, the isotropic lines are circles of zero radius with the centers on the $x$-axe, and the elliptic lines are the circles of imaginary radius.

The main theorem is a corollary of the theorems below. Under the conditions of the theorems to be proven, we will suppose that the polygons $A_{1} A_{2} \ldots A_{n}$ consist of proper points. The extended Lobachevsky plane will play an auxiliary role only.

\section{Smarandache Theorem in Hyperbolic Geometry}

\subsection{The case where the point $M$ is in the proper domain of the Lobachevsky plane}

Theorem 4. Let the points $M_{k}, k=\overline{1, n}$ be orthogonal projections of a point $M$ of the Lobachevsky plane on the sides $A_{k} A_{k+1}\left(A_{n+1}=A_{1}\right)$ of a polygon $P: A_{1} A_{2} \ldots A_{n}$, respectively. Then relation (4) holds, and thus (3) also holds.

$\mathrm{P}$ r o o f. Let us join the point $M$ with the vertices of the polygon $P$ by geodesic segments $A_{1} M, A_{2} M, \ldots, A_{n} M$. Let $a, b$ be the lengthes of the legs, and $c$ be a length of hypotenuse of a rectangular triangle in the hyperbolic plane of curvature $K=-\frac{1}{R^{2}}$, then

$$
\ln \left(\cosh \frac{c}{R}\right)=\ln \left(\cosh \frac{a}{R}\right)+\ln \left(\cosh \frac{b}{R}\right) .
$$

By using (5), we can express the hypotenuses of each of $2 n$ rectangular triangles $A_{k} M_{k} M$ and $A_{k} M_{k-1} M, k=\overline{1, n}, M_{0}=M_{n}$ (among which there may be degenerate ones) by two equalities (see Fig. 1):

$$
\begin{array}{r}
\ln \left(\cosh \frac{A_{k} M}{R}\right)=\ln \left(\cosh \frac{A_{k} M_{k}}{R}\right)+\ln \left(\cosh \frac{M_{k} M}{R}\right) \\
\ln \left(\cosh \frac{A_{k} M}{R}\right)=\ln \left(\cosh \frac{M_{k-1} M}{R}\right)+\ln \left(\cosh \frac{M_{k-1} A_{k}}{R}\right) .
\end{array}
$$

For the case when some of the rectangular triangles get degenerated, the above formulae remain valid. We write the equalities for the right-hand sides 


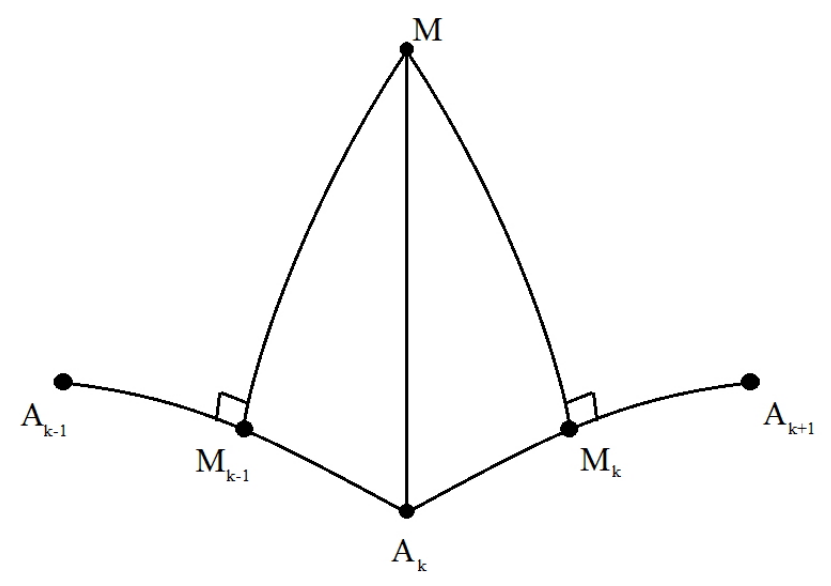

Fig. 1.

and after the summation of $n$ obtained equalities we arrive to the desired relation (4). There are no restrictions to the position of the point $M$ in the Lobachevsky plane (notice that if $M$ coincides with one of the vertices $A_{k}$ of the polygon, then its projections $M_{k-1}$ and $M_{k}$ on the lines $A_{k-1} A_{k}$ and $A_{k} A_{k+1}$, respectively, are considered to coincide with $A_{k}$ ).

\subsection{The case where the perpendiculars to the sides of the polygon intersect at an infinite point $M$ of the Lobachevsky plane}

If the point $M$ is a point at infinity, then the perpendiculars to the sides of the polygon become parallel lines (see Fig. 2). Relation (3) is also valid for this case.

We present the stated above in another form. Remark that any perpendicular to one of the lines passing along the sides of a given polygon $P$ uniquely determines a point $M$ at infinity. Then the theorem is formulated as follows.

Theorem 5. If the perpendiculars to the sides of a polygon $P: A_{1} A_{2} \ldots A_{n}$ at points $M_{k}, 1 \leq k \leq n$, belonging correspondingly to the lines $A_{k} A_{k+1}\left(A_{n+1}=\right.$ $\left.A_{1}\right)$ are parallel lines, then relation (3) holds.

$\mathrm{P} \mathrm{r}$ o o f. This relation can be obtained by a limit passage by moving away a proper point $M_{0}$ in one of the directions to the infinity. Note that $M_{k}^{0}$ denotes the corresponding projections of the point $M_{0}$. Applying the hyperbolic sine theorem, we obtain for all $1 \leq k \leq n$ :

$$
\sinh \frac{M_{k}^{0} M_{0}}{R}=\sin \left(M_{0} A_{k+1} M_{k}^{0}\right) \sinh \frac{A_{k+1} M_{0}}{R},
$$




$$
\sinh \frac{M_{k}^{0} M_{0}}{R}=\sin \left(M_{0} A_{k} M_{k}^{0}\right) \sinh \frac{A_{k} M_{0}}{R} .
$$

We equate the right-hand sides and multiply the $n$ obtained equalities. Since the point $M_{0}$ tends to the infinity, we can suppose that hyperbolic sines in these formulas are not equal to zero, and thus we have the following relation:

$$
\begin{aligned}
& \sin \left(M_{0} A_{1} M_{1}^{0}\right) \sin \left(M_{0} A_{2} M_{2}^{0}\right) \ldots \sin \left(M_{0} A_{n} M_{n}^{0}\right) \\
= & \sin \left(M_{0} A_{2} M_{1}^{0}\right) \sin \left(M_{0} A_{3} M_{2}^{0}\right) \ldots \sin \left(M_{0} A_{1} M_{n}^{0}\right) .
\end{aligned}
$$

Let the point $M_{0}$ tend to the infinity. Then the points $M_{k}^{0}$ converge to the corresponding points $M_{k}$ and the arguments of sines in the last formula become the angles of parallelism of the corresponding segments. Using the known relation $\sin \pi(x)=\frac{1}{\operatorname{ch} \frac{x}{R}}$ between the angle of parallelism and the length of the corresponding perpendicular $[12, \S 2]$, we can get relation (3).

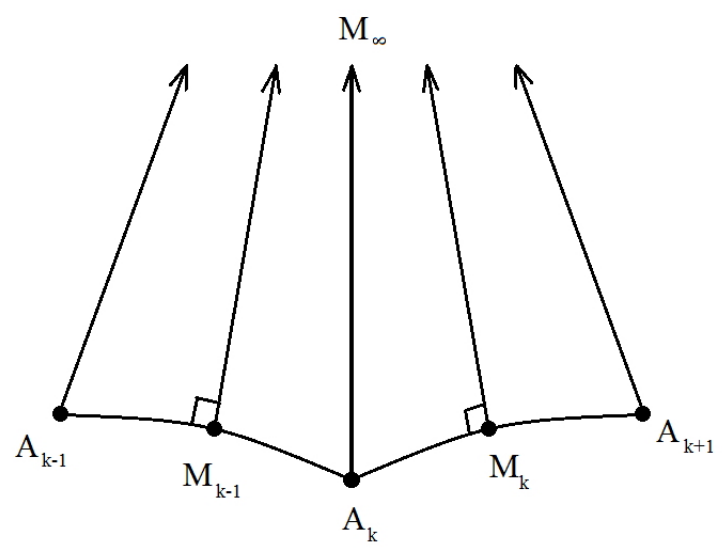

Fig. 2.

Note that the intermediate relation (6) also holds in the Euclidean and spherical geometries.

\subsection{The case where the perpendiculars to the sides of a polygon intersect at an "ideal" point $M$ of the Lobachevsky plane}

We suppose that the points $M_{k}$ are proper points of the Lobachevsky plane. Again, instead of beginning from the projection of the ideal point $M$ (in the same way as in the previous case), we will begin from the bundle of perpendiculars to the sides of the polygon $P$ determining this point. All the lines of the bundle are 
perpendicular to a line of the Lobachevsky plane, that is, to the polar line of the point $M$. The polar line of the point $M$ divergent with all lines passing along the sides of the polygon $P$ uniquely defines the bundle of perpendiculars to the sides of $P$.

Theorem 6. Let the perpendiculars to the sides of a polygon $P: A_{1} A_{2} \ldots A_{n}$ passing through the points $M_{k}, 1 \leq k \leq n$ lying in the lines $A_{k} A_{k+1}\left(A_{n+1}=A_{1}\right)$, respectively, belong to a bundle of divergent lines with the center at an ideal point $M$. Then relation (3) holds.

$\mathrm{P}$ r o o f. The polar line $p$ of the point $M$ is a common perpendicular to the lines $M_{k} M$ and $A_{k} M, 1 \leq k \leq n$. Denote the points of intersection of these lines with the polar $p$ by $M_{k}^{\prime}$ and $\overline{A_{k}^{\prime}}$, respectively. The quadrilaterals $A_{k} M_{k-1} M_{k-1}^{\prime} A_{k}^{\prime}$ and $A_{k} M_{k} M_{k}^{\prime} A_{k}^{\prime}$ have three right angles and acute angles at the vertex $A_{k}$ (see Fig. 3).

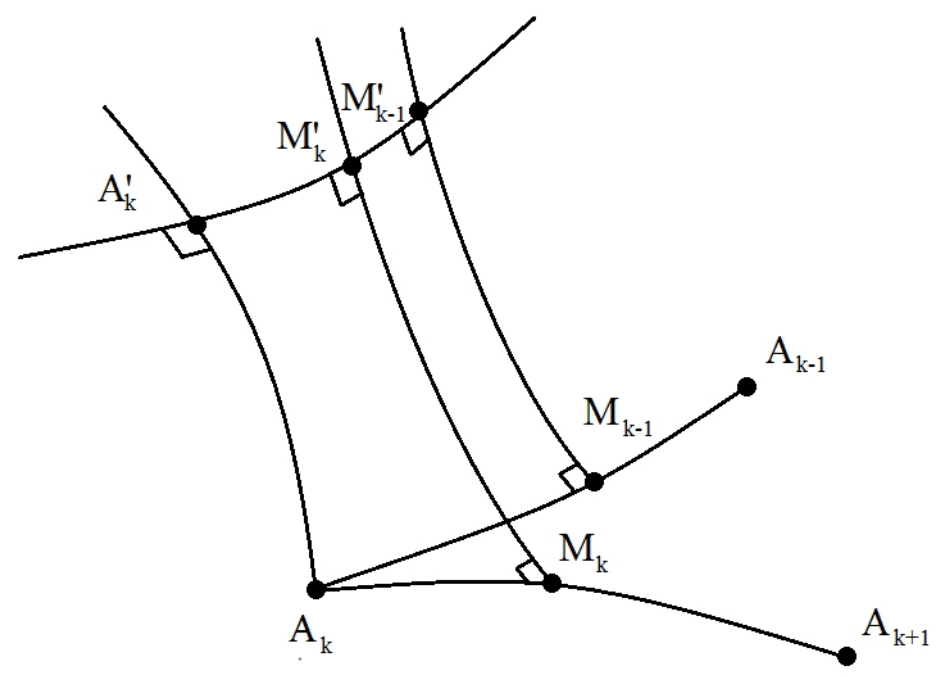

Fig. 3 .

We introduce hyperbolic sines of common base of these quadrilaterals in two forms (see $[12, \S 4])$ :

and

$$
\sinh \frac{A_{k} A_{k}^{\prime}}{R}=\cosh \frac{A_{k} M_{k}}{R} \sinh \frac{M_{k} M_{k}^{\prime}}{R}
$$

$$
\sinh \frac{A_{k} A_{k}^{\prime}}{R}=\cosh \frac{A_{k} M_{k-1}}{R} \sinh \frac{M_{k-1} M_{k-1}^{\prime}}{R} .
$$


Equating the right-hand sides and multiplying the $n$ obtained equalities, we get relation (3). The theorem is proved.

\section{A Partial Inverse of the Main Theorem}

Below we will use the following

$\mathrm{R}$ e $\mathrm{m}$ a $\mathrm{rk}$ 5. Under condition (3), the double inequality

$$
\exp \left(-\frac{A_{1} A_{n}}{R}\right)<\prod_{k=1}^{n-1} \frac{\cosh \frac{A_{k} M_{k}}{R}}{\cosh \frac{A_{k+1} M_{k}}{R}}<\exp \left(\frac{A_{1} A_{n}}{R}\right)
$$

holds for all bundles considered in Theorems 4-6. The similar relations are valid for all sides of the considered polygon.

Theorem 7. Let the perpendiculars to all sides of a polygon $P: A_{1} A_{2} \ldots A_{n}$, except the side $A_{1} A_{n}$, be fixed and belong to one bundle with a center at a proper point, or at a point at infinity, or at a regular ideal point $M$. Let relation (3) hold for a point $M_{n}$ in the line $A_{1} A_{n}$, together with other points $M_{k}, 1 \leq k \leq n-1$. Then the point $M_{n}$ is uniquely determined and the perpendicular to the line $A_{1} A_{n}$ passing through the point $M_{n}$ belongs to the bundle with the center at $M$.

$\mathrm{P}$ r o o f. First, we chose perpendiculars to other sides of the polygon $P: A_{1} A_{2} \ldots A_{n}$ and fix the $n-1$ vertex of the pedal polygon. Then in the leftand right-hand sides of condition (3), two unknown factors remain, each for every side. We find their relation and transform it. By choosing an orientation of the line $A_{1} A_{n}$, we will consider the lengths of geodesic arcs to be relative values, i.e., the values with plus or minus sign in dependence of the chosen direction. The hyperbolic cosine being an even function, its value will not depend on the choice of orientation. We have

$$
\begin{gathered}
\frac{\cosh \frac{A_{1} M_{n}}{R}}{\cosh \frac{A_{n} M_{n}}{R}}=\frac{\cosh \left(\frac{A_{1} A_{n}}{R}+\frac{A_{n} M_{n}}{R}\right)}{\cosh \frac{A_{n} M_{n}}{R}} \\
=\frac{\cosh \frac{A_{1} A_{n}}{R} \cosh \frac{A_{n} M_{n}}{R}+\sinh \frac{A_{1} A_{n}}{R} \sinh \frac{A_{n} M_{n}}{R}}{\cosh \frac{A_{n} M_{n}}{R}} \\
=\cosh \frac{A_{1} A_{n}}{R}+\sinh \frac{A_{1} A_{n}}{R} \tanh \frac{A_{n} M_{n}}{R} .
\end{gathered}
$$

The points $A_{1}$ and $A_{n}$ are fixed. Using the double inequality given in Remark 5 , it is easy to see that for any value of $c \in\left(\exp \left(-\frac{A_{1} A_{n}}{R}\right) ; \exp \left(\frac{A_{1} A_{n}}{R}\right)\right)$, 
there exists a unique point $M_{n}$ in the line $A_{1} A_{n}$ for which the condition

$$
\frac{\cos \frac{A_{1} M_{n}}{R}}{\cos \frac{A_{n} M_{n}}{R}}=c
$$

holds. It completes the proof of Theorem 7 .

In the conditions of Theorem 7 , the choosing of the $(n-2)$ perpendiculars of a bundle with a center at a proper point, or at a point at infinity, or at an ideal point on the Lobachevsky plane is insufficient for finding perpendiculars to the remaining two sides of polygon such that they belong to the same bundle of lines. In other words, the existence of the $(n-2)$ perpendiculars with necessary properties is no sufficient to inverse Theorem 3 (or one of Theorems 4-6).

Indeed, let us take an $n$-gon $P$ for which its $(n-1)$ vertices $A_{1}, A_{2}, \ldots, A_{n-1}$ lie in a circle, or in a horocycle or in an equidistant. Join them by geodesic segments. We can dispose the last vertex $A_{n}$ in the midperpendicular to the segment $A_{1} A_{n-1}$. If the midpoints of all sides of $P$ are chosen as the projections of a point $M$, then relation (3) will be satisfied and the $n-2$ perpendiculars will belong to a bundle with the center $M$ at a proper point, or at a point at infinity, or at an ideal point. Moving the point $A_{n}$ along the midperpendicular to the segment $A_{1} A_{n-1}$, we change the positions of two last perpendiculars. In any of three cases considered, two last perpendiculars can be either intersecting, or parallel, or divergent. The same fact, namely that the fixation of $n-2$ perpendiculars is not sufficient for a partial inversion of Theorems 4-6, can be shown in the following way. We take a polygon with the coinciding vertices $A_{1}$ and $A_{n-1}$. If the $n-2$ perpendiculars to the sides $A_{k} A_{k+1}, 1 \leq k \leq n-2$, belong to one bundle of any type, then the moving of the vertex $A_{n}$ will cause the changing of the position of other perpendiculars. Besides, relation (3) will hold for different choices of the points $M_{n-1}$ and $M_{n}$. In particular, this is true for the case when the points $M_{n-1}$ and $M_{n}$ coincide.

\section{Projection of an Ideal Point to a Hyperbolic Line}

An ideal point $M$ of the extended Lobachevsky plane is called the regular point of the polygon if it is not a pole for any of its sides relatively to the polarity defined by the absolute where the pole of a side is understood as the pole of the line passing through this side. An ideal point $M$, which is the pole of one of the sides of the considered polygon, is called the singular point of the polygon.

Let $M$ be a regular ideal point of the extended Lobachevsky plane for a given polygon. Let us draw the tangent lines from $M$ to the absolute. They divide the projective plane modelling the extended Lobachevsky plane into two parts one of which contains the proper domain. If the poles of all lines containing the sides 
of the polygon lie in this part, then all projections of the point $M$ in the sides of the polygon are proper points.

If the pole of the line containing a side of the polygon lies in the second part, then the projection of the point $M$ in this line is an ideal point. If the pole of one of the lines defined by the sides of the polygon lies in one of the drawn tangents, then the projection of $M$ to this line is a point at infinity, and the line will be parallel to the polar line of the point $M$.

If the ideal point $M$ is the pole of the side $A_{1} A_{n}$ of the polygon $P: A_{1} A_{2} \ldots A_{n}$, then any hyperbolic line of the extended Lobachevsky plane passing through the point $M$ is perpendicular to this side. If we restrict ourselves by the perpendiculars to the sides in the proper domain only, then these perpendiculars cannot intersect at an ideal point singular for the considered polygon. Inversely, if in the ideal domain we take a point $M$ which is the pole of one of the sides of a polygon $P: A_{1} A_{2} \ldots A_{n}$, say, of $A_{1} A_{n}$, then the perpendiculars to all other sides of $P$ will be uniquely defined. Thereby, some projections of the point $M$ to the sides of the polygon $P$ will be ideal points. In particular, the projections to the lines adjacent with the side $A_{1} A_{n}$ and not collinear to it will be obligatorily ideal, and the perpendiculars from $M$ to these sides will be elliptic lines of the ideal domain of the extended Lobachevsky plane. In this case, the distances from the vertices of the pedal polygon to the vertices $A_{1}, A_{2}, \ldots, A_{n}$ will be given by complex numbers.

\section{Some Generalizations and Related Questions}

The theorems proved in the paper can be generalized to the polygons in the three-dimensional Lobachevsky space whose perpendiculars to the sides belong to one of the bundles of spacial lines (intersecting, parallel or divergent).

In the Euclidean plane there is a well-known condition of degeneration of the pedal triangle [13, pp. 40-41]:

The pedal triangle of a point $M$ relatively to the triangle $A B C$ is degenerated, that is, its vertices lie on a line iff the point $M$ belongs to the described circle of the triangle $A B C$.

In the spherical and hyperbolic geometries this result does not hold. It can be shown that in these geometries the pedal triangle of a point $M$ relatively to a triangle $A B C$ is degenerated iff the point $M$ belongs to a curve of degree 3 passing through the vertices of the triangle (the degree of a curve is determined by its equation in projective models of these geometries). It would be interesting to give a characterization of "the curve of degeneration" in dependence of the properties of given triangle in both geometries. 


\section{References}

[1] M. Berger, Geometria, V. 1. Mir, Moscow, 1984. (Russian) (Franch transl.: M. Berger, Géométrie. Espaces eulidiens. CEDIC/Fernand Natan, Paris, 1978.)

[2] F. Smarandache, Problemes avec et Sans ... Problems. pp. 49 \& 54-60, Somi-press, Fes, Morocco, 1983.

[3] A.V. Kostin, Smarandache Theorem in Spherical Geometry. - Izv. Belinsky Penza State Pedagogical University (2012), No. 30, 78-83.

[4] O. Demirel and E. Soyturk, The Hyperbolic Carnot Theorem in the Poincare Disc Model of Hyperbolic Geometry. — Novi Sad J. Math. 38 (2008), 33-39.

[5] C. Barbu and N. Sönmez, On the Carnot Theorem in the Poincare Upper HalfPlane Model of Hyperbolic Geometry. - Acta Universitatis Apulensis 31 (2012), 321-325.

[6] C. Barbu, Contributions to the Study of the Hyperbolic Geometry. PhD thesis Summary. Cluj-Napoca, 2012.

[7] C. Barbu, Smarandache's Pedal Polygon Theorem in the Poincare Disk Model of Hyperbolic Geometry. — Intern. J. Math. Combin. 1 (2010), 99-102.

[8] N. Sönmez and C. Barbu, The Hyperbolic Smarandache Theorem in the Poincare Upper Half-Plane Model of Hyperbolic Geometry. www.IntellectualArchive.com/getfile.phpefile=r1E9bSRUgGH\&orig

[9] N. Sönmez, Request. Personal Communication to I. Sabitov, 28.08.2011.

[10] I.Kh. Sabitov, Solution of Cyclic Polygons. - Mathematic Prosvetshenie 14 (2010), No. 3, 83-106.

[11] B.A. Rozenfeld, Noeuclidean Geometries. Nauka, Moscow, 1969. (Russian)

[12] N.M. Nestorovich, Geometric Constructions in Lobachevsky Plane. GITTL, Moscow-Leningrad, 1951. (Russian)

[13] H.S.M. Coxeter and S.L. Greitzer, Geometry Revisited. Toronto-New York, 1967. (Russ. transl.: G.S.M. Kokseter, S.L. Greitzer, Novye vstrechi s geometriej. Nauka, Moscow, 1978.) 